\title{
]jfis
}

\section{Novel Fuzzy Preview Controller for Rotary Inverted Pendulum under Time Delays}

\author{
Kavirayani Srikanth ${ }^{1}$ and Gundavarapu V Nagesh Kumar ${ }^{2}$ \\ ${ }^{1}$ Department of Electrical and Electronic Engineering, Gayatri Vidya Parishad College of Engineering \\ (Autonomous), Visakhapatnam, India \\ ${ }^{2}$ Department of Electrical and Electronic Engineering, Vignan's Institute of Information Technology, \\ Visakhapatnam, India
}

\begin{abstract}
A novel fuzzy preview controller based on look up tables is proposed for control design of a rotary inverted pendulum represented with integrated time delay in system matrices. The proposed control achieved control of all system states with predefined standard requirements. The advantage of preview control helps in conservation of energy as the control input acts upon after a lookup making the system robust even under the impact of custom designed system time delays that were incorporated into the system. The proposed method shows the influence of time delay can be countered effectively by integrating the delay into the system matrix and then using the novel fuzzy granular preview control.
\end{abstract}

Keywords: Time delay, Preview control, Rotary inverted pendulum, Granular computing

\section{Introduction}

The rotary inverted pendulum has been involved as the test bed in the control domain for many years, the reason being the mechanism of having a rotary servo controlling the pendulum over the upright equilibrium position which is making the system a complex dynamic plant which becomes an example in control education. The applications of the system have been seen in literature in mapping the logic applied to the control problem in the fields of humanoid robot walking, gesture control, segway transport, satellite launch and other potential areas in which the model can be evolved into a handy tool for various defense and military applications.

The rotary inverted pendulum has a control problem has been investigated by researchers

cCThis is an Open Access article distributed under the terms of the Creative Commons Attribution Non-Commercial License (http://creativecommons.org/licenses/ by-nc/3.0// which permits unrestricted noncommercial use, distribution, and reproduction in any medium, provided the original work is properly cited. earlier in various capacities of studies on normal controllability, observability of states to design of linear quadratic controllers. Jadlovska and Sarnovsky [1] have studies using the state dependent algebraic ricatti equation as in [1] where comparative analysis was done with other classical techniques. Srikanth and Kumar [2] have applied the condition of time delay to the state space model by taking a integrated model involving the states and time delay and have shown the stability margin using particle swarm optimization. Lhee et al. [3] have designed a fuzzy logic controller similar to an sliding mode control considering with dead zone parameters, however the time delay is not integrated into the system dynamic model directly. Birla and Swarup [4] have investigated the case of a preview based controller only for a linear inverted pendulum based on evolutionary algorithms.

The analysis of the system with time delay integrated into the system is not found much in 
the literature. Srikanth and Kumar [2, 5] have developed dynamic models with integration of the time delay parameter for double and rotary inverted pendulum models which is the plant that is considered here. Daswon [6] have proposed a method to deal with variable time delays. Sun et al. [7] have presented a state dependent stabilization criterion and Benitez-Perez and Garcia-Nocetti [8] have indicated a method for deterministic time delay in control configuration. The novelty being considered here with preview or lookup of the values is efficient as control actions are taken after a preview is not found in literature and is being proposed here. Tria et al. [9] in their paper discusses about a variable control law to improve the dynamic behavior of the wind turbine system with sliding mode control as the control approach with emphasis on a fuzzy regulator design for the active and reactive powers. Mohiuddin [10] has discussed about the modelling and simulation for speed control of a DC motor using fuzzy logic with comparison for conventional PID controllers and fuzzy logic controllers using chopper circuit and proved fuzzy logic controllers outperform conventional controllers. Chakraborty et al. [11] have discussed about the swingup and control of the inverted pendulum system with a fuzzy logic controller by developing the mathematical modeling and performing the analysis using a fuzzy logic controller and have compared the performance of fuzzy logic controller to traditional PID controller in terms of system specifications of time delay, peak overshoot and have proved that the overshoots and settling time are less with fuzzy logic controllers which is a required observation. Zhang and Liu [12] elaborated on the fuzzy logic techniques and the way the technique can be applied to dynamic system analysis. Wu et al. [13] discussed time delay systems and how time delay plays a crucial role in the design and control of systems and it's studies. Lai and Chiang [14] have discussed about the granular computing technique where scaling down information into granules would help with the better study of a system in general and how it could be used in conjunction with fuzzy logic for improvising system studies. Driankov et al. [15] have discussed about the basic aspects of fuzzy control and how it could be extrapolated and tested for large scale systems. Zhang et al. [16] discusses about fuzzy sets based decision logic language proposed for granular computing that have extended predication logic language based on intuitionist fuzzy sets. Rubio-Solis et al. [17] integrate a few machine learning methods such as Granular Computing $(\mathrm{GrC})$, RBF Neural Networks (RBF-NN), Feature Selection (FS) to create a DDFM framework which is sensitive to imbalanced data. Wang and Pedrycz [18] show that the robust granular optimiza- tion solutions exhibit a stronger resistance to the uncertainty which is an important point for consideration of the granular computing technique. Petrosino and Pal [19] show that the granular computing is a primary constituent of soft computing whose objective is to provide flexible information processing capability for handling real-life ambiguous situations which is another important reason for choosing the application of this technique. Pedrycz et al. [20] have elaborated on the granular mapping which is a important aspect in fuzzy control.

This paper proposes a new method of controlling the rotary inverted pendulum with a novel fuzzy preview controller with granular computing which is efficient in terms of energy usage. The proposed model considered is generated by row and column generation for incorporating the time delay component into the system definition and then a lookup based fuzzy control is applied to the plant model which gives a better control in specifications of peak overshoots, settling time when compared to the real time performance observed over a Quanser rotary inverted pendulum controlled with pole placement with fixed poles.

\section{Mathematical Model}

The state model defined in Eqs. (3) and (4) is obtained from [2] using the basic dynamic equations defined in Eqs. (1) and (2).

$$
\begin{aligned}
& M_{0} \ddot{\theta}_{0}+M_{1} \theta_{1} \cos \theta_{0} \ddot{\theta}_{1}-M_{2} \sin \theta_{0} \cos \theta_{0} \dot{\theta}_{1}^{2} \\
& \quad-M_{3} g \sin \theta_{0} \\
& =0, \\
& M_{1} \cos \theta_{0} \ddot{\theta}_{1}+M_{4}+\left(M_{4} \sin ^{2} \theta_{0}\right) \ddot{\theta}_{1}-M_{1} \sin \theta_{0} \ddot{\theta}_{0}^{2} \\
& \quad+2 M_{2} \sin \theta_{0} \cos \theta_{0} \dot{\theta}_{0} \dot{\theta}_{1} \\
& =\tau,
\end{aligned}
$$

where $\tau$ in Eq. (2) refers to the control input which is applied to the shaft of the arm and $M_{i}(i=0,1,2,3,4)$ in Eqs. (1) and (2) are positive system parameters defined as

$$
\begin{aligned}
& M_{0}=I_{1}+l_{1}^{2} m_{1}, \\
& M_{1}=m_{1} l_{1} L_{2}, \\
& M_{2}=l_{1}^{2} m_{1}, \\
& M_{3}=l_{1} m_{1}, \\
& M_{4}=I_{2}+l_{2}^{2} m_{2}+L_{2}^{2} m_{1} .
\end{aligned}
$$

The mathematical model for the rotary inverted pendulum is taken directly as in [2] which is an integrated model of the 


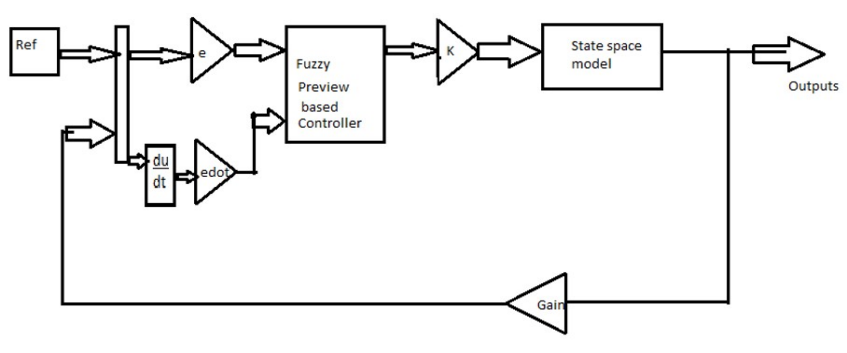

Figure 1. System model with fuzzy control.

system with time delay given by the generic form of

$$
\dot{X}=A X+B U
$$

where $X$ representing 5 states for the translation and rotation of the arm and the pendulum with 4 states that represent the system model by Eqs. (8) and 9). One additional state that represents the time delay is integrated into the system. The model is reconfigured with the delay embedded in order to make the system a minimum state variable model which makes the unified representation easier and decoupling the system into various canonical forms easier. The output equation is

$$
Y=C X+D U
$$

The fuzzy control block diagram model that is proposed is represented in Figure 1.

As shown in Figure 1, the important parameters that are playing a key role are the error and the error rate which represent two inputs to the fuzzy preview controller which lookups the values based on which a decision is made and the output is passed on to the controller for amplification of the signal which is then passed as input to the plant. The feedback path has a LQR controller which does the state feedback control. The fuzzy granular based preview controller has a faster rule explosion which results in efficient control.

A weighted average method is adopted to calculate the hierarchical fuzzy controller with type-1 fuzzy controller and type- 2 fuzzy controller.

$$
u=w_{\text {type1 }} * u_{\text {type1fuzzy }}+w_{\text {type2 }} * u_{\text {type2fuzzy }} .
$$

As given in Eq. [10], where granulation is causing refinement in the way the control effort is used for the smoother control of the plant model. The incremental control action is not only a function of error and rate of error but also the time delay component which makes it a nonlinear controller which is more efficient than a liberalized LQR controller in terms of the control effort for reduced oscillations.

\section{Results}

The system matrices representing the model of the integrated rotary inverted pendulum model that were taken during the process are given as follows taken from [2] are as follows:

$$
\begin{aligned}
A & =\left[\begin{array}{ccccc}
0 & 0 & 1 & 0 & 0 \\
0 & 0 & 0 & 1 & 0 \\
0 & 39.2 & -14.52 & 0 & 0 \\
0 & 81.78 & -13.98 & 0 & 0 \\
-4 / \text { Tuo } & 2 / \text { Tuo } & 0 & 0 & 0
\end{array}\right], \\
B & =\left[\begin{array}{c}
0 \\
0 \\
25.54 \\
24.59 \\
0
\end{array}\right]
\end{aligned}
$$

$A$ is an identity matrix and $B$ is a null matrix for the model of the Rotary pendulum taken from [2]. The system definitions were obtained by values taken from Appendix A. The system is as such obtained from the dynamic model of the rotary inverted pendulum with an integration of the time delay component added to the system as a fifth state other than the four states which define the vertical and horizontal motions of the plant. The plant model has two rotations $(\alpha, \theta)$ which are varied in order to achieve the control. The velocity components of the same were as well considered in the system. The system model dynamics are developed from a standard Quanser product of the rotary inverted pendulum as taken in [2].

The gain values that have been calculated from the classical linear quadratic controller are given as Gain Matrix = [-101.0896175.5642 - 20.172523.46261.3030];

The initial conditions for all the cases is taken as $X_{o}=$ [pi/60000];

It is assumed that the initial conditions for the pendulum assign the pendulum to a position where it is in the vicinity of the upright equilibrium position ensuring that the case studies only the stabilization about the upright equilibrium position and not the control problem of swing up from the downward equilibrium position which is another case study found in literature.

The various test cases tested as per the block diagram represented in Figure 1 is shown below where the analysis is done for Cases 1 to 12. The error values variations resulted in control 
Table 1. Test case of error variations

\begin{tabular}{ccccc}
\hline Case ID & E & Edot & Standard ref & Control action \\
\hline 1 & 20 & .002 & .001 & Success \\
2 & 2 & .002 & .001 & Success \\
3 & 2 & 0.2 & .001 & Fails \\
4 & 2 & .002 & .001 & Fails \\
5 & 2 & .2 & .001 & Fails \\
6 & 2 & .02 & 1 & Fails \\
7 & 2 & .002 & 1 & Success \\
8 & .002 & .002 & 1 & Fails \\
9 & .002 & .00002 & 1 & Fails \\
10 & .002 & .00002 & .001 & Fails \\
11 & 20 & 20 & .001 & Fails \\
12 & 2000 & 20 & .001 & Fails \\
13 & 2000 & 2000 & 1 & Fails \\
\hline
\end{tabular}

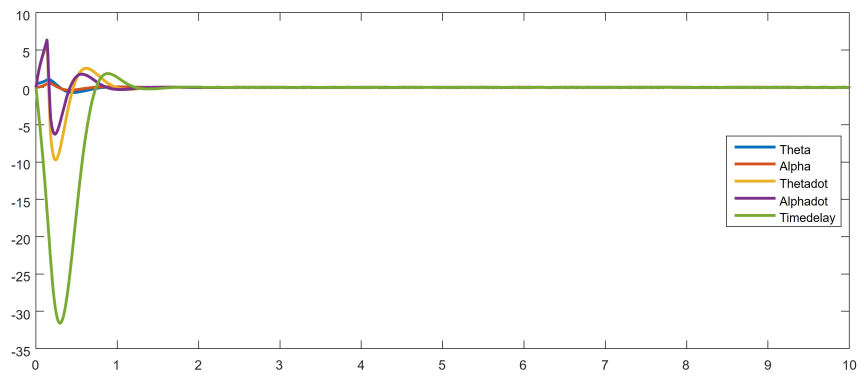

Figure 2. Case 1 output states.

action where the pendulum was stabilized about the upright equilibrium position.

The various plots that have been obtained are given below in Figures 2 and 3. In Figure 2, the control effort was successful in the stabilization of the pendulum and Figure 3 we present a counter example case where the failure of the stabilization occurs which is indicative that the failure is also possible if the constraints on the system definition limits are violated. The fuzzy lookup table surface plot is as shown below in Figure 4. Table 1 indicates the rule explosion taking the two inputs of error and the derivative of error. The two values are passed in order to have a faster rule explosion when compared with the case of taking only the rule. The result clearly shows the feasibility of solution using the lookup definitions made which act as the rule base.

It can be observed clearly the status of control for the system from swing up to upright equilibrium in Figures 5 and 6 where

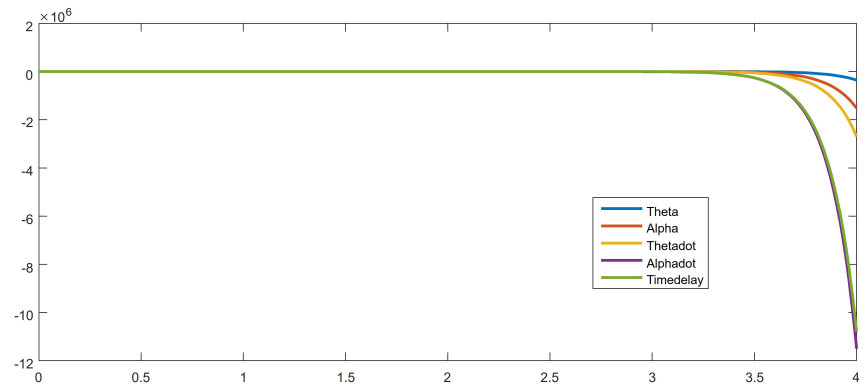

Figure 3. Case 9 of output states (unstable).

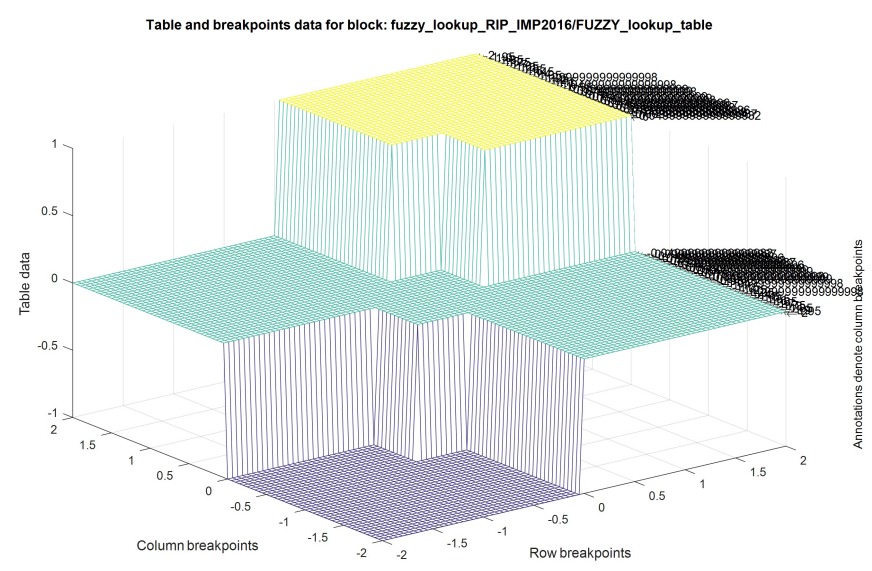

Figure 4. Lookup table classification.

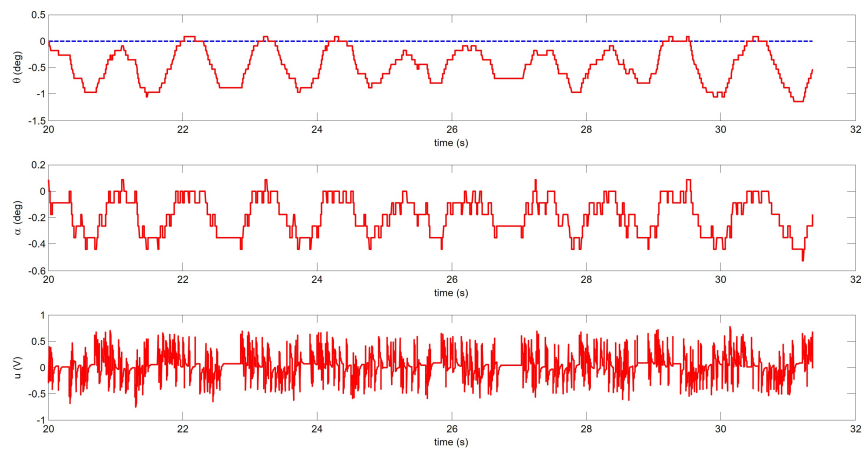

Figure 5. Typical real time performance of RIP with offset.

the switching happens between stable and unstable regions for the laboratory model of the Quanser rotary inverted pendulum which is an actual experiment conducted on the test bed.

Figure 7 indicates the performance of the RIP in real time on an actual experiment conducted and comparing this with Figure 2 we can clearly see that the peak overshoots are reduced and the settling time are improved. 


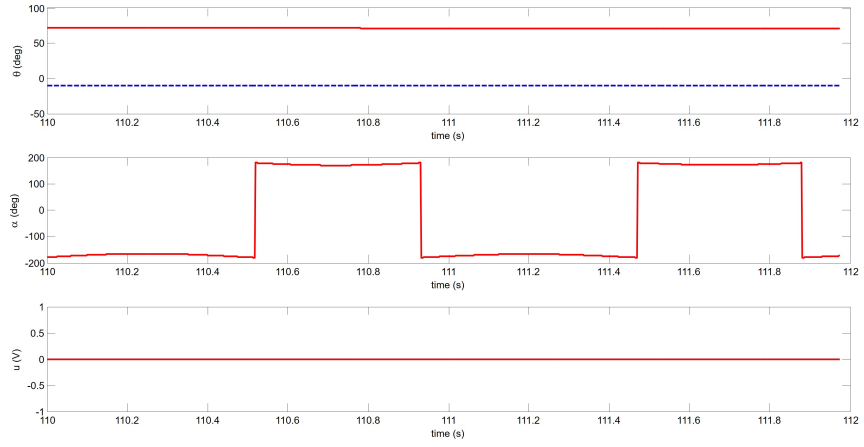

Figure 6. Typical stabilization failure case in real time.

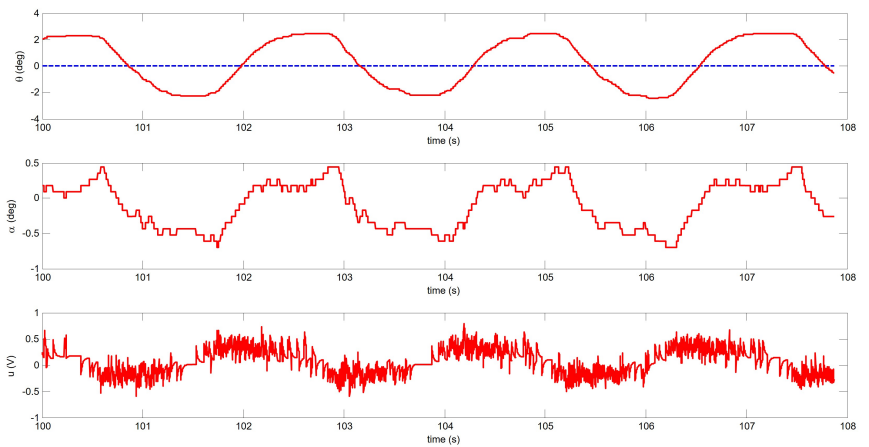

Figure 7. Swing up and stabilization in real time [2].

\section{Conclusions}

The analysis on the rotary inverted pendulum has clearly indicated that the stabilization about upright equilibrium could be achieved with the preview based fuzzy controller with granular computing. This can be used for the analysis of high end dynamic systems wherein the control action required can be achieved by preview first which is an efficient method when compared to other methods. The reason a preview control is better when compared with other methods is it minimizes the computation time involved in the process as a preview would guide the system faster to convergence than a system without preview.

\section{Acknowledgements}

The authors would like to thank the management of Gayatri Vidya Parishad College of Engineering (Autonomous), and GITAM University for providing the necessary research facilities. We thank RGUKT, Nuzvid, INDIA for conduct of experiments on the real-time test bed of the rotary inverted pendulum.

\section{Appendix A. System Parameters of Rotary Pen- dulum}

\begin{tabular}{|c|c|c|}
\hline Parameter & Description & Value \\
\hline $\mathbf{M}_{p}$ & Mass of pendulum & $0.127 \mathrm{~kg}$ \\
\hline $\mathrm{M}_{r}$ & Mass of rotary arm & $0.2570 \mathrm{~kg}$ \\
\hline $\mathrm{R}_{m}$ & $\begin{array}{l}\text { Motor armature } \\
\text { resistance }\end{array}$ & $2.6 \mathrm{ohm}$ \\
\hline $\mathrm{B}_{p}$ & $\begin{array}{l}\text { Equivalent viscous } \\
\text { damping coefficient }\end{array}$ & $\begin{array}{l}0.0024 \\
\text { N.m.s/rad }\end{array}$ \\
\hline $\mathbf{J}_{e q}$ & $\begin{array}{l}\text { Equivalent moment of } \\
\text { inertia w.r.to load }\end{array}$ & $0.0021 \mathrm{~kg} \cdot \mathrm{m}^{2}$ \\
\hline $\mathbf{J}_{m}$ & $\begin{array}{l}\text { Motor armature } \\
\text { moment of inertia }\end{array}$ & $\begin{array}{l}4.6063 \mathrm{e}-07 \\
\mathrm{~kg} \cdot \mathrm{m}^{2}\end{array}$ \\
\hline $\mathbf{J}_{r}$ & $\begin{array}{l}\text { Rotary arm moment of } \\
\text { Inertia }\end{array}$ & $\begin{array}{l}9.9829 \mathrm{e}-04 \\
\mathrm{~kg} \cdot \mathrm{m}^{2}\end{array}$ \\
\hline $\mathrm{L}_{p}$ & Pendulum full length & $0.3365 \mathrm{~m}$ \\
\hline $\mathrm{K}_{m}$ & $\begin{array}{l}\text { Motor back-emf } \\
\text { constant }\end{array}$ & 0.0077 V.s/rd \\
\hline $\mathrm{K}_{t}$ & Motor torque constant & 0.0077 N.m/A \\
\hline $1_{p}$ & $\begin{array}{l}\text { Distance from pivot to } \\
\text { centre of gravity }\end{array}$ & $0.1556 \mathrm{mt}$ \\
\hline $1_{r}$ & $\begin{array}{l}\text { Rotary arm distance } \\
\text { from pivot to center of } \\
\text { mass }\end{array}$ & $0.0619 \mathrm{mt}$ \\
\hline VMAX_AMP & $\begin{array}{l}\text { Amplifier maximum } \\
\text { output voltage }\end{array}$ & $24 \mathrm{~V}$ \\
\hline IMAX_AMP & $\begin{array}{l}\text { Amplifier maximum } \\
\text { output current }\end{array}$ & $4 \mathrm{~A}$ \\
\hline
\end{tabular}

\section{References}

[1] S. Jadlovska and J. Sarnovsky, "Application of the state dependent Riccati equation method in nonlinear control design for inverted pendulum systems," in Proceedings of the IEEE 11th International Symposium on Intelligent Systems and Informatics, Serbia, 2013, pp. 209-214. http: //dx.doi.org/10.1109/SISY.2013.6662572

[2] K. Srikanth and G. V. N. Kumar, "Rotary inverted pendulum control and the impact of time delay on switching between stable and unstable states with enhanced particle swarm optimization," International Journal of Computer and Communication System Engineering, vol. 2, no. 4, pp. 569-574, 2015. 
[3] C. G. Lhee, J. S. Park, H. S. Ahn, and D. H. Kim, "Sliding mode-like fuzzy logic control with self-tuning the dead zone parameters," IEEE Transactions on Fuzzy Systems, vol. 9, no. 2, pp. 343-348, 2001. http://dx.doi.org/10.1109/ 91.919255

[4] N. Birla and A. Swarup, "Performance of preview control based on evolutionary algorithms," International Journal of Advanced Science and Technology, vol. 38, pp. 37-52, 2012.

[5] K. Srikanth and G. N. Kumar, "Stabilization at upright equilibrium position of a double inverted pendulum with unconstrained bat optimization," International Journal of Computational Sciences and Applications, vol. 5, no. 5, pp. 87-101, 2015. http://dx.doi.org/10.5121/ijcsa.2015.5508

[6] J. G. Dawson, "Fuzzy logic control of linear systems with variable time delay," in Proceedings of the 9th IEEE International Symposium on Intelligent Control, Columbus, OH, 1994, pp. 5-10. http://dx.doi.org/10.1109/ISIC.1994. 367850

[7] X. Sun, H. Zhang, and Y. Wang, "Stabilization algorithm for interval type-2 TSK fuzzy logic control systems with bounded time-varying delay," in Proceedings of 34th Chinese Control Conference (CCC), Hangzhou, 2015, pp. 321-325. http://dx.doi.org/10.1109/ChiCC.2015.7259657

[8] H. Benitez-Perez and F. Garcia-Nocetti, "Switching fuzzy logic control for a reconfigurable system considering communication time delays," in Proceedings of European Control Conference (ECC), Cambridge, UK, 2003, pp. 1435-1440.

[9] F. Z. Tria, K. Srairi, M. T. Benchouia, B. Mahdad, and M. E. H. Benbouzid, "An hybrid control based on fuzzy logic and a second order sliding mode for MPPT in wind energy conversion systems," International Journal on Electrical Engineering and Informatics, vol. 8, no. 4, pp. 711-726, 2016. https://doi.org/10.15676/ijeei.2016.8.4.1

[10] M. S. Mohiuddin, "Performance comparison of conventional controller with fuzzy logic controller using chopper circuit and fuzzy tuned PID controller," Indonesian Journal of Electrical Engineering and Informatics, vol. 2, no. 4, pp. 189-200, 2014. https://doi.org/10.11591/ijeei.v2i4. 120
[11] K. Chakraborty, A. K. Sen, R. D. Basak, and R. Raushan, "Case study of various parameters by applying swing up control for inverted pendulum," Indonesian Journal of Electrical Engineering and Informatics, vol. 3, no. 3, pp. 167-171, 2015. https://doi.org/10.11591/ijeei.v3i3.148

[12] H. Zhang and D. Liu, Fuzzy Modeling and Fuzzy Control. Boston, MA: Springer, 2006.

[13] M. Wu, Y. He, and J. H. She, Stability Analysis and Robust Control of Time-Delay Systems. Beijing: Springer Science Press, 2010.

[14] R. Lai and D. Chiang, "Constraint-based granular computing for fuzzy modeling," in Proceedings of the 2002 IEEE International Conference on Fuzzy Systems, Honolulu, HI, 2002, pp. 584-589. https://doi.org/10.1109/FUZZ.2002. 1005057

[15] D. Driankov, H. Hellendoorn, and M. Reinfrank, An Introduction to Fuzzy Control. New Delhi: Narosa Publishing House, 2013.

[16] Y. Zhang, X. Zhu, and Z. Huang, "Fuzzy sets based granular logics for granular computing," in Proceedings of International Conference on Computational Intelligence and Software Engineering, Wuhan, China, 2009, pp. 1-6. https://doi.org/10.1109/CISE.2009.5363975

[17] A. Rubio-Solis, G. Panoutsos, and S. Thornton, "A datadriven fuzzy modelling framework for the classification of imbalanced data," in Proceedings of IEEE 8th International Conference on Intelligent Systems (IS), Sofia, Bulgaria, 2016, pp. 302-307. https://doi.org/10.1109/IS. 2016.7737438

[18] S. Wang and W. Pedrycz, "Robust granular optimization: a structured approach for optimization under integrated uncertainty," IEEE Transactions on Fuzzy Systems, vol. 23, no. 5, pp. 1372-1386, 2015. https://doi.org/10.1109/ TFUZZ.2014.2360941

[19] A. Petrosino and S. K. Pal, "Guest editorial on decision making in human and machine vision," IEEE Transactions on Systems, Man, and Cybernetics: Systems, vol. 44, no. 5, pp. 521-522, 2014. https://doi.org/10.1109/TSMC.2014. 2313155

[20] W. Pedrycz, R. Al-Hmouz, A. Morfeq, and A. Balamash, "The design of free structure granular mappings: the use of 
the principle of justifiable granularity," IEEE Transactions on Cybernetics, vol. 43, no. 6, pp. 2105-2113, 2013. https: //doi.org/10.1109/TCYB.2013.2240384

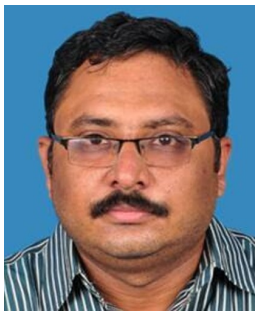

Kavirayani Srikanth was born in Visakhapatna, India. He obtained his Master of Science in electrical engineering from University of Missouri-Columbia, USA, specializing in intelligent systems. He is currently a part-time research scholar pursuing doctoral program in GITAM, Visakhapatnam, India. He holds the position of assistant professor in electrical and electronics engineering in Gayatri Vidya Parishad College of Engineering (Autonomous), Visakhapatnam, India. His research interests include intelligent systems, robotics, control and automation. He has to his credit over 10 journals and nearly 20 conference publications.

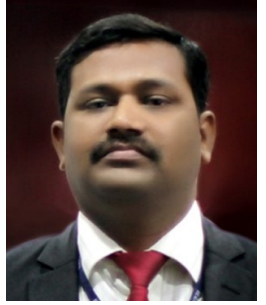

Gundavarapu V. Nagesh Kumar was born in Visakhapatnam, India, in 1977. He received his B.E. degree from GITAM, Visakhapatnam, and his M.E. degree from Andhra University, Visakhapatnam. He received his doctoral degree from JNT University, Hyderabad. He is currently working as a Professor in the Department ofElectrical and Electronic Engineering, Vignan's Institute of Information Technology, Visakhapatnam. His research interests include evolutionary computation and FACTS devices. 